\title{
Identification of Memory Kernels in Heat Flow Measuring Heat Flux at the Ends of the Bar*
}

\author{
E. Pais \\ Institute of Mathematics at Tallinn UT \\ Ehitajate rd 5, 19086 Tallinn, Estonia \\ E-mail: ennopais@stv.ee
}

Received December 4, 2009; revised March 15, 2010; published online November 15, 2010

\begin{abstract}
An inverse problem to determine time- and space-dependent relaxation kernels of internal energy and heat flux with first kind boundary conditions by means of heat flux measurements is considered. The case when observations of the heat flux are made at the ends of the bar with thermal memory was not studied before. Existence and uniqueness of a solution to the inverse problem are proved.
\end{abstract}

Keywords: inverse problem, memory kernel, heat flow.

AMS Subject Classification: 35R30; 80A23.

\section{Introduction}

In the linear theory of heat conduction in materials with thermal memory appear two memory (or relaxation) kernels $[1,2,7,8]$. To determine these unknown kernels the corresponding inverse problems are used.

The memory kernels can be degenerate, i.e. represented as finite sums of products of known space-dependent functions times unknown time-dependent coefficients. This is so when either the medium is piecewise continuous or a problem for a general kernel is replaced by a related problem for an approximated kernel. The unknown coefficients are recovered by a finite number of measurements of certain time-dependent characteristics of the solution of the direct problem. Inverse problems of such a type were studied in $[3,4,5,6]$, $[9,10,11,12]$. The papers $[9,10,11,12]$ treat an inverse problem for material with memory having two relaxation kernels: the kernels of internal energy and heat flux. As additional information were used the measurements of the temperature or the heat flux in finite number of interior points of the unit bar.

In the present paper, for the first time is studied the same inverse problem for two relaxation kernels using additional data at the ends of the bar. As now

\footnotetext{
* The financial support of Estonian Science Foundation is gratefully acknowledged (Grant nr. 7728).
} 
the additional information consists only of two measurements we must restrict us to the case of two unknown functions: one for each memory kernel. So we can not consider here the general case of degenerate memory kernels. The main difference is that system of equations in the Laplace domain contains now the transform of a fractional derivative of one of unknown functions. Also we need to find some new estimates of the Green function in this case. The existence and uniqueness are proved for the inverse problem with the first kind boundary conditions and the heat flux observations. The problem with the third kind boundary conditions can be treated in a similar manner but some other estimates of the Green function must be used.

In Section 2 we formulate the direct and inverse problems and in Section 3 apply the Laplace transform to them. In Section 5 we rewrite the transformed problems in the fixed-point form. Section 4 contains the estimates of the Green function and Section 6 describes results for the direct problem. Main existence and uniqueness results for the inverse problem are presented in Section 7 of the paper.

\section{Direct and Inverse Problem}

We consider the heat flow in a rigid non-homogeneous bar consisting of a material with thermal memory. In the linear approximation the process is described by the following integro-differential equation of heat conduction

$$
\begin{array}{r}
\beta(x) \frac{\partial}{\partial t} u(x, t)+\frac{\partial}{\partial t} \int_{0}^{t} n(x, t-\tau) u(x, \tau) d \tau=\frac{\partial}{\partial x}\left(\lambda(x) u_{x}(x, t)\right) \\
-\frac{\partial}{\partial x} \int_{0}^{t} m(x, t-\tau) u_{x}(x, \tau) d \tau+r(x, t), \quad x \in(0,1), \quad t>0 .
\end{array}
$$

Here $u$ is the temperature of the bar, $\beta$ the heat capacity, $\lambda$ the heat conduction and $r$ the heat supply. The integral terms contain a memory kernel of the internal energy $n$ and a memory kernel of the heat flux $m$.

The solution of the equation (2.1) satisfies the initial condition

$$
u(x, 0)=\varphi(x), \quad x \in(0,1)
$$

and either the the boundary conditions of the first kind

$$
u(0, t)=f_{1}(t), \quad u(1, t)=f_{2}(t), \quad t>0
$$

or the boundary conditions of the third kind

$$
-q(0, t)=\alpha_{1}\left(u(0, t)-f_{1}(t)\right), \quad q(1, t)=\alpha_{2}\left(u(1, t)-f_{2}(t)\right), \quad t>0,
$$

where $q(x, t)$ is the heat flux, $\varphi$ and $f_{j}, j=1,2$ are given functions, $\alpha_{j}, j=1,2$ given constants. The heat flux is given by equality

$$
q(x, t)=-\lambda(x) u_{x}(x, t)+\int_{0}^{t} m(x, t-\tau) u_{x}(x, \tau) d \tau, \quad x \in(0,1) .
$$


Equation (2.1) with the initial condition (2.2) and boundary conditions (2.3) or (2.4) form the direct problem for the temperature $u$.

Inverse problem has for goal to determine the memory kernels $n$ and $m$. We consider here kernels having form of products

$$
n(x, t)=\nu(x) n(t), \quad m(x, t)=\mu(x) m(t),
$$

where $\nu(x)$ and $\mu(x)$ are given functions and $n(t)$, and $m(t)$ are unknown time-dependent coefficients. To recover the functions $n(t)$, and $m(t)$ we use additional data in form of the observations at the ends of the bar. We can measure the temperature

$$
u(0, t)=h_{1}(t), \quad u(1, t)=h_{2}(t), \quad t>0
$$

or the heat flux

$$
\begin{aligned}
& q(0, t)=-\lambda(0) u_{x}(0, t)+\int_{0}^{t} \mu(0) m(t-\tau) u_{x}(0, \tau) d \tau=h_{1}(t) \\
& q(1, t)=-\lambda(1) u_{x}(1, t)+\int_{0}^{t} \mu(1) m(t-\tau) u_{x}(1, \tau) d \tau=h_{2}(t), \quad t>0 .
\end{aligned}
$$

In case of the first kind boundary conditions only the heat flux observations can be used, since temperatures are already used as boundary conditions. In case of the third kind boundary conditions we have relation between the temperature and the heat flux at the ends of the bar. So the temperature observation enable us to find the heat flux and vice versa. As a result all three inverse problems use the same data: temperature and heat flux values at the ends of the bar. For this reason the inverse problems are similar and are described by similar equations. We will treat the problem with the first kind boundary conditions and the heat flux observations which is given by the relations (2.1)-(2.3), (2.5), (2.6).

\section{Problem in Laplace Domain}

Applying the Laplace transform to the equation (2.1) we obtain

$$
\begin{array}{r}
\beta(x)[p U(x, p)-\varphi(x)]+p N(p) \nu(x) U(x, p)=\frac{\partial}{\partial x}\left(\lambda(x) U_{x}(x, p)\right) \\
-M(p) \frac{\partial}{\partial x}\left(\mu(x) U_{x}(x, p)\right)+R(x, p),
\end{array}
$$

where $N=\mathcal{L} n_{j}, M=\mathcal{L} m_{k}, R=\mathcal{L} r$,

$$
U(x, p)=\mathcal{L} u(x, t)=\int_{0}^{\infty} e^{-p t} u(x, t) d t, \quad \operatorname{Re} p>\sigma .
$$

The boundary conditions (2.3) are given by

$$
U(0, p)=F_{1}(p), \quad U(1, p)=F_{2}(p), \quad \operatorname{Re} p>\sigma,
$$


where $F_{j}=\mathcal{L} f_{j}, j=1,2$ and the additional conditions (2.6) get the form

$$
\begin{aligned}
& (-\lambda(0)+M(p) \mu(0)) U_{x}(0, p)=H_{1}(p), \\
& (-\lambda(1)+M(p) \mu(1)) U_{x}(1, p)=H_{2}(p)
\end{aligned}
$$

with $H_{j}(p)=\mathcal{L} h_{i}, j=1,2$.

We represent the equation (3.1) in the form

$$
\begin{aligned}
(L U)(x, p)=p N(p) \nu(x) U(x, p) & +M(p) \frac{\partial}{\partial x}\left(\mu(x) U_{x}(x, p)\right) \\
& -R(x, p)-\beta(x) \varphi(x),
\end{aligned}
$$

where

$$
(L U)(x, p)=\frac{\partial}{\partial x}\left(\lambda(x) U_{x}(x, p)\right)-\beta(x) p U(x, p), \quad x \in(0,1)
$$

is the differential operator. We introduce the Green function of operator $L$ with homogeneous first kind boundary conditions, i.e.

$$
\begin{array}{ll}
L_{y} G(x, y, p)=\delta(x, y), & x \in(0,1), y \in(0,1), \\
G(x, 0, p)=G(x, 1, p)=0, & x \in(0,1) .
\end{array}
$$

Here $L_{y}$ is the operator $L$ with respect to the variable $y$.

The solution of (3.3) is given as

$$
\begin{aligned}
U(x, p) & =p N(p) \int_{0}^{1} G(x, y, p) \nu(y) U(y, p) d y \\
& +M(p) \int_{0}^{1} G(x, y, p) \frac{\partial}{\partial y}\left(\mu(y) U_{y}(y, p)\right) d y-\Phi(x, p),
\end{aligned}
$$

where

$$
\begin{aligned}
\Phi(x, p) & =\int_{0}^{1} G(x, y, p)[\beta(y) \varphi(y)+R(y, p)] d y \\
& +\lambda(0) G_{y}(x, 0, p) F_{1}(p)-\lambda(1) G_{y}(x, 1, p) F_{2}(p) .
\end{aligned}
$$

Integrating the second integral in (3.5) by parts and observing (3.4) we have

$$
\begin{aligned}
U(x, p) & =N(p) \int_{0}^{1} p G(x, y, p) \nu(y) U(y, p) d y \\
& -M(p) \int_{0}^{1} G_{y}(x, y, p) \mu(y) U_{y}(y, p) d y-\Phi(x, p) .
\end{aligned}
$$

Differentiating (3.6) with respect to $x$ we obtain the equation for $U_{x}(x, p)$

$$
\begin{aligned}
U_{x}(x, p)= & N(p) \int_{0}^{1} p G_{x}(x, y, p) \nu(y) U(y, p) d y \\
& +M(p) \int_{0}^{1} G_{x}(x, y, p) \frac{\partial}{\partial y}\left(\mu(y) U_{y}(y, p)\right) d y-\Phi_{x}(x, p) .
\end{aligned}
$$


We split the second integral into two parts, from 0 to $x$ and from $x$ to 1 , and integrate them by parts. From boundary conditions we have

$$
G_{x}(x, 0, p)=G_{x}(x, 1, p)=0, \quad 0<x<1 .
$$

Using the jump relation (see [13], p. 169)

$$
G_{x}(x, x-0, p)-G_{x}(x, x+0, p)=\frac{1}{\lambda(x)}, \quad 0<x<1
$$

we get

$$
\begin{aligned}
U_{x}(x, p) & =\frac{1}{\lambda(x)} M(p) \mu(x) U_{x}(x, p)+N(p) \int_{0}^{1} p G_{x}(x, y, p) \nu(y) U(y, p) d y \\
& -M_{k}(p) \int_{0}^{1} G_{x y}(x, y, p) \mu(y) U_{y}(y, p) d y-\Phi_{x}(x, p) .
\end{aligned}
$$

(3.6) and (3.7) form a system of integral equations for the direct problem.

\section{Functional Spaces and Estimation of Green Function}

To analyse the direct and inverse problems we define the spaces

$$
\mathcal{A}_{\gamma, \sigma}=\left\{V: V(p) \text { is holomorphic on } \operatorname{Re} p>\sigma,\|V\|_{\gamma, \sigma}<\infty\right\},
$$

where $\|V\|_{\gamma, \sigma}=\sup _{\operatorname{Re} p>\sigma}|p|^{\gamma}|V(p)|$ and

$$
\left(\mathcal{A}_{\gamma, \sigma}\right)^{K}=\left\{V=\left(V_{1}, \ldots, V_{N}\right): V_{k}(p) \in \mathcal{A}_{\gamma, \sigma}, k=1, \ldots, K\right\}
$$

with the norm $\|V\|_{\gamma, \sigma}=\sum_{k=1}^{K}\left\|V_{k}\right\|_{\gamma, \sigma}$.

We note that $\mathcal{A}_{\gamma, \sigma} \subset \mathcal{A}_{\gamma, \sigma^{\prime}},\left(\mathcal{A}_{\gamma, \sigma}\right)^{K} \subset\left(\mathcal{A}_{\gamma, \sigma^{\prime}}\right)^{K}$ and $\|\cdot\|_{\gamma, \sigma^{\prime}} \leq\|\cdot\|_{\gamma, \sigma}$ if $\sigma^{\prime}>\sigma$.

Let $\alpha$ be a real number such that $1<\alpha<\frac{3}{2}$. Moreover, let $c=\left(c_{1}, \ldots, c_{K}\right)$ be a given vector. We will introduce the spaces

$$
\mathcal{M}_{c, \alpha, \sigma}=\left\{Z: Z=\frac{c}{p}+V(p), V \in\left(\mathcal{A}_{\alpha, \sigma}\right)^{K}\right\}
$$

of vectors $Z=\left(Z_{1}, \ldots, Z_{K}\right)$ and the Banach spaces of $x$ - and $p$-dependent functions

$$
\mathcal{B}_{\gamma, \sigma}=\left\{F(x, p): F(x, \cdot) \in \mathcal{A}_{\gamma, \sigma} \text { for } x \in[0,1], F(\cdot, p) \in C[0,1] \text { for } \operatorname{Re} p>\sigma\right\}
$$

with the norms

$$
\|F\|_{\gamma, \sigma}=\max _{0 \leq x \leq 1} \sup _{\operatorname{Re} p>\sigma}|p|^{\gamma}|F(x, p)| .
$$

We need also the Banach spaces for pairs of functions $B=\left(B_{1}, B_{2}\right)$. These are spaces $\mathcal{B}_{\sigma}=\mathcal{B}_{\alpha, \sigma} \times \mathcal{B}_{\alpha-\frac{1}{2}, \sigma}$ with the norm

$$
\|B\|_{\sigma}=\left\|B_{1}\right\|_{\alpha, \sigma}+\left\|B_{2}\right\|_{\alpha-\frac{1}{2}, \sigma} .
$$


We have following first-order asymptotics $[3,5,9,10,12]$ for the Green function and its derivatives

$$
\begin{array}{r}
G(x, y, p)=\frac{1}{d_{0}(p)} \frac{1}{a(x) a(y)} \frac{1}{s} \begin{cases}\operatorname{sh} s z \cdot \operatorname{sh} s(1-w)+O_{1} & \text { for } x \leq y \\
\operatorname{sh} s w \cdot \operatorname{sh} s(1-z)+O_{2} & \text { for } y \leq x\end{cases} \\
G_{x}(x, y, p)=\frac{1}{l d_{0}(p)} \frac{b(x)}{a(y)} \begin{cases}\operatorname{ch} s z \cdot \operatorname{sh} s(1-w)+O_{3} & \text { for } x \leq y \\
\operatorname{sh} c w \cdot \operatorname{ch} s(1-z)+O_{4} & \text { for } y \leq x\end{cases} \\
G_{x y}(x, y, p)=\frac{b(x) b(y)}{l^{2} d_{0}(p)} s\left\{\begin{array}{lll}
\operatorname{ch} s z \cdot \operatorname{ch} s(1-w)+O_{5} & \text { for } x \leq y \\
\operatorname{ch} s w \cdot \operatorname{ch} s(1-z)+O_{6} & \text { for } y \leq x .
\end{array}\right.
\end{array}
$$

Here $s=l \sqrt{p}, d_{0}(p)=\frac{1}{l} \operatorname{sh} s+O\left(\frac{e^{s}}{s}\right), a(x)=\sqrt[4]{\beta(x) \lambda(x)}, b(x)=\beta^{1 / 4} \lambda^{-3 / 4}$

$$
z=\frac{1}{l} \int_{0}^{x} \sqrt{\frac{\beta(\eta)}{\lambda(\eta)}} d \eta, w=\frac{1}{l} \int_{0}^{y} \sqrt{\frac{\beta(\eta)}{\lambda(\eta)}} d \eta, l=\int_{0}^{1} \sqrt{\frac{\beta(\eta)}{\lambda(\eta)}} d \eta
$$

and

$$
O_{1}=O\left(\frac{e^{s(1-w+z)}}{s}\right), \quad O_{2}=O\left(\frac{e^{s(w+1-z)}}{s}\right),
$$

for $\operatorname{Re} p \rightarrow+\infty$ holding uniformly in $x$ and $y$ from $[0,1]$ and $\operatorname{Im} p \in \mathbb{R}$. $O_{3}$ and $\mathrm{O}_{5}$ behave like $\mathrm{O}_{1}$ and $\mathrm{O}_{4}, \mathrm{O}_{6}$ like $\mathrm{O}_{2}$.

Estimates of the Green function are given in the following lemmas.

Lemma 1. Let $\lambda, \beta \in C^{2}[0,1]$ and $\lambda, \beta>0$ in $[0,1]$. Then

$$
\begin{aligned}
& C_{1}=\sup _{\substack{0 \leq x \leq 1 \\
\operatorname{Re} p>0}}|p| \int_{0}^{1}|G(x, y, p)| d y<\infty, \\
& C_{2}=\sup _{\substack{0 \leq x \leq 1 \\
\operatorname{Re} p>0}} \sqrt{|p|} \int_{0}^{1}\left|G_{x}(x, y, p)\right| d y<\infty, \\
& C_{3}=\sup _{\substack{0 \leq x \leq 1 \\
\operatorname{Re} p>0}} \sqrt{|p|} \int_{0}^{1}\left|G_{y}(x, y, p)\right| d y<\infty, \\
& C_{4}=\sup _{\substack{0 \leq x \leq 1 \\
\operatorname{Re} p>0}} \int_{0}^{1}\left|G_{x y}(x, y, p)\right| d y<\infty .
\end{aligned}
$$

Moreover,

$$
\begin{aligned}
& \sqrt{|p|}|G(x, 0, p)| \leq \bar{C}_{1} e^{-\bar{C}_{2} \sqrt{|p|} x} \quad \forall x \in[0,1], \operatorname{Re} p>0, \\
& \sqrt{|p|}|G(x, 1, p)| \leq \bar{C}_{3} e^{-\bar{C}_{4} \sqrt{|p|}(1-x)} \quad \forall x \in[0,1], \quad \operatorname{Re} p>0,
\end{aligned}
$$

where $\bar{C}_{1}, \bar{C}_{2}, \bar{C}_{3}, \bar{C}_{4}$ are certain positive constants.

The assertions $(4.2)-(4.5)$ were proved in $[3,5]$, the assertions $(4.6)$ in [12] 
Lemma 2. Let $\lambda, \beta \in C^{2}[0,1], \lambda, \beta>0$ in $[0,1]$ and $v \in C^{1}[0,1]$. Then for $k=1,2$

$$
\sup _{\operatorname{Re} p>0}\left|\sqrt{|p|}\left(\int_{0}^{1} \sqrt{p} G_{x}\left(x_{k}, y, p\right) v(y) d y-\frac{(-1)^{k} v\left(x_{k}\right)}{\sqrt{\lambda\left(x_{k}\right) \beta\left(x_{k}\right)}}\right)\right| \leq C_{5}\|v\|_{C^{1}[0,1]},
$$

where $x_{1}=0, x_{2}=1$ and $C_{6}$ is independent of $x$.

Proof. From asymptotic representation (4.1) of the derivative $G_{x}$ we have

$$
\begin{aligned}
& G_{x}(0, y, p)=\frac{1}{l d_{0}(p)} b(0)\left[\frac{1}{a(y)} \operatorname{sh} s(w-1)+O\left(\frac{e^{s(1-w)}}{s}\right)\right], \\
& G_{x}(1, y, p)=\frac{1}{l d_{0}(p)} b(1)\left[\frac{1}{a(y)} \operatorname{sh} s w+O\left(\frac{e^{s w}}{s}\right)\right] .
\end{aligned}
$$

As $\sqrt{p}=s / l$ we get

$$
\int_{0}^{1} \sqrt{p} G_{x}(0, y, p) v(y) d y=\frac{s b(0)}{l^{2} d_{0}(p)} \int_{0}^{1} \frac{v(y)}{a(y)} \frac{1}{s w^{\prime}(y)} \frac{\partial}{\partial y} \operatorname{ch} s(w-1) d y+\mathcal{O}\left(\frac{1}{s}\right) .
$$

Since $d_{0}(p)$ has the asymptotic $l d_{0}(p)=\operatorname{sh} s+\mathcal{O}\left(\frac{e^{s w}}{s}\right)$ we derive for the last term

$$
\begin{aligned}
\left|\frac{1}{l d_{0}(p)} \int_{0}^{1} e^{s(1-w)} d y\right| & =\left|\frac{1}{l d_{0}(p)} \frac{1}{s} \int_{0}^{1} \frac{1}{w^{\prime}(y)} \frac{\partial}{\partial y} e^{s(1-w)} d y\right| \\
& \leq \frac{C}{s}\left|\frac{1}{l d_{0}(p)} e^{s(1-w)}\right|_{0}^{1} \mid=\frac{C}{s} \frac{1-e^{s}}{\operatorname{sh} s+\mathcal{O}\left(e^{s} / s\right)}=\mathcal{O}\left(\frac{1}{s}\right)
\end{aligned}
$$

Integrating by parts and observing the equality $\frac{1}{a(y) w^{\prime}(y)}=l \bar{b}(y)$ we obtain

$$
\begin{aligned}
& \int_{0}^{1} \sqrt{p} G_{x}(0, y, p) v(y) d y=\frac{b(0)}{l d_{0}(p)}\left[\left.\bar{b}(y) v(y) \operatorname{ch} s(w-1)\right|_{0} ^{1}\right. \\
& \left.-\int_{0}^{1}(\bar{b}(y) v(y))^{\prime} \operatorname{ch} s(w-1) d y\right]+\mathcal{O}\left(\frac{1}{s}\right)=\frac{b(0)}{l d_{0}(p)}[\bar{b}(1) v(1)-\bar{b}(0) v(0) \operatorname{ch} s] \\
& +\mathcal{O}\left(\frac{1}{s}\right)=\frac{b(0)}{\operatorname{sh} s+\mathcal{O}\left(\frac{e^{s}}{s}\right)}[\bar{b}(1) v(1)-\bar{b}(0) v(0) \operatorname{ch} s]+\mathcal{O}\left(\frac{1}{s}\right) \\
& =-v(0) / \sqrt{\lambda(0) \beta(0)}+\mathcal{O}(1 / s) .
\end{aligned}
$$

Here $b(0) \bar{b}(0)=1 / a(0)^{2}=1 / \sqrt{\lambda(0) \beta(0)}$. Similarly we have

$$
\begin{aligned}
& \int_{0}^{1} \sqrt{p} G_{x}(1, y, p) v(y) d y=\frac{b(1)}{l d_{0}(p)}\left[\left.\bar{b}(y) v(y) \operatorname{ch} s w\right|_{0} ^{1}-\int_{0}^{1}(\bar{b}(y) v(y))^{\prime} \operatorname{ch} s w d y\right] \\
& +\mathcal{O}\left(\frac{1}{s}\right)=\frac{b(0)}{l d_{0}(p)}[\bar{b}(1) v(1) \operatorname{ch} s-\bar{b}(0) v(0)]+\mathcal{O}\left(\frac{1}{s}\right)=\frac{v(1)}{\sqrt{\lambda(1) \beta(1)}}+\mathcal{O}\left(\frac{1}{s}\right) .
\end{aligned}
$$

The lemma is proved. 
Lemma 3. Let $\lambda, \beta \in C^{2}[0,1], \lambda, \beta>0$ in $[0,1]$ and $v \in C^{1}[0,1]$. Then

$$
\sup _{\operatorname{Re} p>0}\left|\sqrt{|p|}\left(\int_{0}^{1} G_{x y}\left(x_{k}, y, p\right) v(y) d y-\frac{v\left(x_{k}\right)}{\lambda\left(x_{k}\right)}\right)\right| \leq C_{6}\|v\|_{C^{1}[0,1]},
$$

where $x_{1}=0, x_{2}=1$ and $C_{6}$ is independent of $x$.

Lemma 3 was proved in $[3,5]$.

\section{Fixed-Point System for the Inverse Problem}

We deduce here a fixed-point system for the inverse problem in the Laplace domain. Using additional conditions (3.2) we obtain from (3.7)

$$
\begin{gathered}
N(p) \int_{0}^{1} p G_{x}\left(x_{k}, y, p\right) \nu(y) U(y, p) d y-M(p) \int_{0}^{1} G_{x y}\left(x_{k}, y, p\right) \mu(y) U_{y}(y, p) d y \\
=\Phi_{x}\left(x_{k}, p\right)-\frac{H_{k}(p)}{\lambda\left(x_{k}\right)}, \quad k=1,2 ; \quad x_{1}=0, x_{2}=1 .
\end{gathered}
$$

Multiplying the both sides by $p$ and separating the principal part of the equation we have

$$
\begin{aligned}
\sqrt{p} N & (p) \frac{\nu\left(x_{k}\right) \varphi\left(x_{k}\right)}{\sqrt{\lambda\left(x_{k}\right) \beta\left(x_{k}\right)}}+M(p) \frac{\mu\left(x_{k}\right) \varphi^{\prime}\left(x_{k}\right)}{\lambda\left(x_{k}\right)} \\
& =\sqrt{p} N(p) \int_{0}^{1} \sqrt{p} G_{x}\left(x_{k}, y, p\right) \nu(y)[p U(y, p)-\varphi(y)] d y \\
& -M(p) \int_{0}^{1} p G_{x y}\left(x_{k}, y, p\right) \mu(y)\left[p U_{y}(y, p)-\varphi^{\prime}(y)\right] d y \\
& +\sqrt{p} N(p)\left[\int_{0}^{1} \sqrt{p} G_{x}\left(x_{k}, y, p\right) \nu(y) \varphi(y) d y+\frac{\nu\left(x_{k}\right) \varphi\left(x_{k}\right)}{\sqrt{\lambda\left(x_{k}\right) \beta\left(x_{k}\right)}}\right] \\
& -M(p)\left[\int_{0}^{1} G_{x y}\left(x_{k}, y, p\right) \mu(y) \varphi^{\prime}(y) d y-\frac{\mu\left(x_{k}\right) \varphi^{\prime}\left(x_{k}\right)}{\lambda\left(x_{k}\right)}\right] \\
& -p\left[\Phi_{x}\left(x_{k}, p\right)-\frac{H_{k}(p)}{\lambda\left(x_{k}\right)}\right]=\mathcal{F}_{k}(N, M), \quad k=1,2 ; \quad x_{1}=0, x_{2}=1 .
\end{aligned}
$$

Here

$$
\begin{aligned}
\Phi_{x}\left(x_{k}, p\right) & =\int_{0}^{1} G_{x}\left(x_{k}, y, p\right)[\beta(y) \varphi(y)+R(y, p)] d y+\lambda(0) G_{x y}\left(x_{k}, 0, p\right) F_{1}(p) \\
& -\lambda(1) G_{x y}\left(x_{k}, 1, p\right) F_{2}(p), \quad x_{1}=0, x_{2}=1 .
\end{aligned}
$$

We will write this system in a matrix form $\Gamma Z=\mathcal{F}(Z)$ introducing matrix

$$
\Gamma=\left(\begin{array}{cc}
\frac{\nu(0) \varphi(0)}{\sqrt{\lambda(0) \beta(0)}} & \frac{\mu(0) \varphi^{\prime}(0)}{\lambda(0)} \\
-\frac{\nu(1) \varphi(1)}{\sqrt{\lambda(1) \beta(1)}} & \frac{\mu(1) \varphi^{\prime}(1)}{\lambda(1)}
\end{array}\right)
$$


and vectors

$$
Z=\left(\begin{array}{r}
\sqrt{p} N(p) \\
M(p)
\end{array}\right), \quad \mathcal{F}(Z)=\left(\begin{array}{c}
\mathcal{F}_{1}(Z) \\
\mathcal{F}_{2}(Z)
\end{array}\right)
$$

The product $\sqrt{p} N(p)$ is the image by Laplace transform of a half-order derivative of the function $n(t), \mathcal{L} \frac{d^{\frac{1}{2}}}{d t^{\frac{1}{2}}} n(t)=\sqrt{p} N(p)$, where this derivative is given by

$$
\frac{d^{\frac{1}{2}}}{d t^{\frac{1}{2}}} n(t)=\frac{d}{d t} \frac{1}{\Gamma\left(\frac{1}{2}\right)} \int_{0}^{t} \frac{n(\tau)}{\sqrt{t-\tau}} d \tau .
$$

We introduce the functions

$$
B_{1}[Z](x, p)=p U[Z](x, p)-\varphi(x), \quad B_{2}[Z](x, p)=p U_{x}[Z](x, p)-\varphi^{\prime}(x),
$$

where $U[Z](x, p)$ is the solution of $(5.1),(5.2)$ with the given vector $Z$.

Now we can rewrite the system in the fixed-point form

$$
Z=\Gamma^{-1} \mathcal{F}(Z)
$$

where

$$
\begin{aligned}
\mathcal{F}_{k}[Z](p) & =\sqrt{p} N(p)\left[\int_{0}^{1} \sqrt{p} G_{x}\left(x_{k}, y, p\right) \nu(y) B_{1}[Z](y, p) d y\right. \\
& \left.+\int_{0}^{1} \sqrt{p} G_{x}\left(x_{k}, y, p\right) \nu(y) \varphi(y) d y+\frac{\nu\left(x_{k}\right) \varphi\left(x_{k}\right)}{\sqrt{\lambda\left(x_{k}\right) \beta\left(x_{k}\right)}}\right] \\
& -M(p)\left[-\int_{0}^{1} G_{x y}\left(x_{k}, y, p\right) \mu(y) B_{2}[Z](y, p) d y\right. \\
& \left.+\int_{0}^{1} G_{x y}\left(x_{k}, y, p\right) \mu(y) \varphi^{\prime}(y) d y-\frac{\mu\left(x_{k}\right) \varphi^{\prime}\left(x_{k}\right)}{\lambda\left(x_{k}\right)}\right]+\Psi_{k}(p), k=1,2 \\
\Psi_{k}(p) & =-p\left[\Phi_{x}\left(x_{k}, p\right)-\frac{H_{k}(p)}{\lambda\left(x_{k}\right)}\right], \quad x_{1}=0, x_{2}=1
\end{aligned}
$$

We form also equations for $B_{1}[Z](x, p)$ and $B_{2}[Z](x, p)$ rewriting the system (3.6), (3.7) in terms of these functions

$$
\begin{aligned}
& B_{1}[Z](x, p)=\sqrt{p} N(p) \int_{0}^{1} \sqrt{p} G(x, y, p) \nu(y)\left[B_{1}[Z](y, p)+\varphi(y)\right] d y \\
& -M(p) \int_{0}^{1} G_{y}(x, y, p) \mu(y)\left[B_{2}[Z](y, p)+\varphi^{\prime}(y)\right] d y-\Phi(x, p)-\varphi(x), \\
& B_{2}[Z](x, p)=\sqrt{p} N(p) \int_{0}^{1} \sqrt{p} G_{x}(x, y, p) \nu(y)\left[B_{1}[Z](y, p)+\varphi(y)\right] d y \\
& +M(p)\left\{\frac{\mu(x)}{\lambda(x)} B_{2}[Z](x, p)-\int_{0}^{1} G_{x y}(x, y, p) \mu(y)\left[B_{2}[Z](y, p)+\varphi^{\prime}(y)\right] d y\right\} \\
& +M(p) \frac{\mu(x) \varphi^{\prime}(x)}{\lambda(x)}-\Phi_{x}(x, p)-\varphi^{\prime}(x) .
\end{aligned}
$$


We obtain the following fixed-point equation for the vector

$$
B[Z]=A[Z] B[Z]+b[Z]
$$

where $A[Z]=\left(A_{1}[Z], A_{2}[Z]\right)$ is the $Z$-dependent linear operator of $B$ with the components

$$
\begin{aligned}
\left(A_{1}[Z] B\right)(x, p) & =\sqrt{p} N(p) \int_{0}^{1} \sqrt{p} G(x, y, p) \nu(y) B_{1}(y, p) d y \\
& -M(p) \int_{0}^{1} G_{y}(x, y, p) \mu(y) B_{2}(y, p) d y \\
\left(A_{2}[Z] B\right)(x, p) & =\sqrt{p} N(p) \int_{0}^{1} \sqrt{p} G_{x}(x, y, p) \nu(y) B_{1}(y, p) d y \\
& +M(p)\left[\frac{\mu(x)}{\lambda(x)} B_{2}(x, p)-\int_{0}^{1} G_{x y}(x, y, p) \mu(y) B_{2}(y, p) d y\right]
\end{aligned}
$$

and $b[Z]=\left(b_{1}[Z], b_{2}[Z]\right)$ is the $Z$-dependent $B$-free term with the components

$$
\begin{aligned}
b_{1}[Z](x, p) & =\sqrt{p} N(p) \int_{0}^{1} \sqrt{p} G(x, y, p) \nu(y) \varphi(y) d y \\
& -M(p) \int_{0}^{1} G_{y}(x, y, p) \mu(y) \varphi^{\prime}(y) d y-\Phi(x, p)-\varphi(x), \\
b_{2}[Z](x, p) & =\sqrt{p} N(p) \int_{0}^{1} \sqrt{p} G_{x}(x, y, p) \nu(y) \varphi(y) d y \\
& +M(p)\left[\frac{\mu(x) \varphi^{\prime}(x)}{\lambda(x)}-\int_{0}^{1} G_{x y}(x, y, p) \mu(y) \varphi^{\prime}(y) d y\right]-\Phi_{x}(x, p)-\varphi^{\prime}(x) .
\end{aligned}
$$

\section{Analysis of Direct Problem}

Here we study the equation (5.7) under assumptions

$$
\left.\begin{array}{l}
\lambda, \beta \in C^{2}[0,1], \quad \lambda, \beta>0 \\
\Phi(x) \text { and } \Phi_{x}(x) \text { belong to } \mathcal{B}_{1, \sigma_{0}} \text { and } \mathcal{B}_{\frac{1}{2}, \sigma_{0}} \\
\text { respectively with some } \sigma_{0} \geq 0 ; \\
\nu(x) \in C[0,1], \quad \mu(x) \in C[0,1], \quad \varphi(x) \in C^{1}[0,1] .
\end{array}\right\}
$$

Lemma 4. Let the assumptions (6.1) hold. If $Z=c / p+V \in \mathcal{M}_{c, \alpha, \sigma}$ then the vector function $b[Z]$, given by (5.9), belongs to $\mathcal{B}_{\sigma_{0}}$ and satisfies the estimate

$$
\|b[Z]\|_{\sigma} \leq \operatorname{Const}\left(1+\frac{|c|}{\sqrt{\sigma}}+\frac{\|V\|_{\alpha, \sigma}}{\sigma^{\alpha-\frac{1}{2}}}\right)
$$

with any $\sigma \geq \sigma_{0}$, where $|c|=\left|c_{1}\right|+\left|c_{2}\right|$. Moreover, for every $\sigma \geq \sigma_{0}$ and $Z_{1}=c / p+V^{1}, Z_{2}=c / p+V^{2} \in \mathcal{M}_{c, \alpha, \sigma}$ the difference of the vector $b[Z]$ fulfils the estimate

$$
\left\|b\left[Z_{1}\right]-b\left[Z_{2}\right]\right\|_{\sigma} \leq \text { Const } \frac{1}{\sigma^{\alpha-\frac{1}{2}}}\left\|V^{1}-V^{2}\right\|_{\alpha, \sigma}
$$


with some constant.

Proof. Taking $Z=c / p+V$ in (5.9) and multiplying by $|p|$ we have

$$
\begin{aligned}
|p|\left|b_{1}[Z](x, p)\right| \leq \frac{1}{\sqrt{p}}\left(\left|c_{1}\right|+\frac{|p|^{\alpha}\left|V_{1}(p)\right|}{|p|^{\alpha-1}}\right)|p| \int_{0}^{1}|G(x, y, p)| d y\|\nu \cdot \varphi\|_{C[0,1]} \\
\quad+\frac{1}{\sqrt{p}}\left(\left|c_{2}\right|+\frac{|p|^{\alpha}\left|V_{2}(p)\right|}{|p|^{\alpha-1}}\right) \sqrt{|p|} \int_{0}^{1}\left|G_{y}(x, y, p)\right| d y\left\|\mu \cdot \varphi^{\prime}\right\|_{C[0,1]} \\
\quad+|p||\Phi(x, p)+\varphi(x)| .
\end{aligned}
$$

Using the assertions (4.2), (4.4) of Lemma 1 and the definitions of the norms $\|\cdot\|_{\gamma, \sigma},\|\cdot\|_{\sigma}$ we obtain

$$
\begin{aligned}
\left\|b_{1}[Z]\right\|_{1, \sigma} & \leq\left(\left|c_{1}\right|+\frac{\left\|V_{1}\right\|_{\alpha, \sigma}}{\sigma^{\alpha-1}}\right) \frac{C_{1}}{\sqrt{\sigma}}\|\nu \cdot \varphi\|_{C[0,1]} \\
& +\left(\left|c_{2}\right|+\frac{\left\|V_{2}\right\|_{\alpha, \sigma}}{\sigma^{\alpha-1}}\right) \frac{C_{3}}{\sqrt{\sigma}}\left\|\mu \cdot \varphi^{\prime}\right\|_{C[0,1]}+\|\Phi+\varphi\|_{1, \sigma_{0}}
\end{aligned}
$$

for $\operatorname{Re} p>\sigma, \sigma \geq \sigma_{0}, x \in[0,1]$. We get

$$
\left\|b_{1}[Z]\right\|_{1, \sigma} \leq \frac{\text { Const }}{\sqrt{\sigma}}\left(|c|+\frac{\|V\|_{\alpha, \sigma}}{\sigma^{\alpha-1}}\right)+\|\Phi+\varphi\|_{1, \sigma_{0}}, \quad \sigma \geq \sigma_{0}
$$

with some constant. Performing similar transformations with $b_{2}[Z]$ we have

$$
\begin{aligned}
\sqrt{|p|}\left|b_{2}[Z](x, p)\right| & \leq \frac{1}{\sqrt{|p|}}\left(\left|c_{1}\right|+\frac{|p|^{\alpha}\left|V_{1}(p)\right|}{|p|^{\alpha-1}}\right) \sqrt{|p|} \int_{0}^{1}\left|G_{x}(x, y, p)\right| d y \\
& \times\|\nu \cdot \varphi\|_{C[0,1]}+\frac{1}{\sqrt{|p|}}\left(\left|c_{2}\right|+\frac{|p|^{\alpha}\left|V_{2}(p)\right|}{|p|^{\alpha-1}}\right)\left[\left|\frac{\mu(x) \varphi^{\prime}(x)}{\lambda(x)}\right|\right. \\
& \left.+\int_{0}^{1}\left|G_{x y}(x, y, p)\right| d y\left\|\mu \cdot \varphi^{\prime}\right\|\right]+\sqrt{|p|}\left|\Phi_{x}(x, p)+\varphi^{\prime}(x)\right|, \\
\left\|b_{2}[Z]\right\|_{\frac{1}{2}, \sigma} & \leq\left(\left|c_{1}\right|+\frac{\left\|V_{1}\right\|_{\alpha, \sigma}}{\sigma^{\alpha-1}}\right) \frac{C_{2}}{\sqrt{\sigma}}\|\nu \cdot \varphi\|_{C[0,1]}+\frac{1}{\sqrt{\sigma}}\left(\left|c_{2}\right|+\frac{\left\|V_{2}\right\|_{\alpha, \sigma}}{\sigma^{\alpha-1}}\right) \\
& \times\left(\frac{1}{\lambda_{0}}+C_{4}\right)\left\|\mu \cdot \varphi^{\prime}\right\|_{C[0,1]}+\left\|\Phi_{x}+\varphi^{\prime}\right\|_{\frac{1}{2}, \sigma_{0}}
\end{aligned}
$$

for $\sigma \geq \sigma_{0}$, where $\lambda_{0}:=\min _{0 \leq x \leq 1} \lambda(x)>0$. And we get

$$
\left\|b_{2}[Z]\right\|_{\frac{1}{2}, \sigma} \leq \frac{\text { Const }}{\sqrt{\sigma}}\left(|c|+\frac{\|V\|_{\alpha, \sigma}}{\sigma^{\alpha-1}}\right)+\left\|\Phi_{x}+\varphi^{\prime}\right\|_{\frac{1}{2}, \sigma_{0}}, \quad \sigma \geq \sigma_{0} .
$$

Inequalities (6.4) and (6.5) yield $b[Z]=\left(b_{1}[Z], b_{2}[Z]\right) \in \mathcal{B}_{\sigma}$ for $\sigma \geq \sigma_{0}$ and the estimate $(6.3)$. To prove $(6.2)$ we take $Z=Z_{1}-Z_{2}$. Then the components of the vector $b[Z]=b\left[Z_{1}\right]-b\left[Z_{2}\right]$ are expressed by the formulas $(5.9)$ with $\Phi(x)+\varphi(x)=0$. Using the estimates (6.4) and (6.5) for the components of $b[Z]$ and observing that $Z_{1}-Z_{2}=V^{1}-V^{2}$ we deduce (6.3). The lemma is proved. 
Lemma 5. Let the assumptions (6.1) hold. If $Z=c / p+V \in \mathcal{M}_{c, \alpha, \sigma}$ then the linear operator $A[Z]$, defined by (5.8) is well-defined and bounded in $\mathcal{B}_{\sigma}$ and satisfies the estimate

$$
\|A[Z]\|_{\mathscr{L}\left(\mathcal{B}_{\sigma}\right)} \leq C_{0}\left[|c| / \sigma^{\frac{3}{2}}+\|V\|_{\alpha, \sigma} / \sigma^{\alpha+\frac{1}{2}}\right]
$$

for any $\sigma \geq \sigma_{0}$ with a constant $C_{0}$. Moreover, taking $Z_{1}=c / p+V^{1}, Z_{2}=$ $c / p+V^{2} \in \mathcal{M}_{c, \alpha, \sigma}$, the estimate for difference

$$
\left\|\left(A\left[Z_{1}\right]-A\left[Z_{2}\right]\right)\right\|_{\mathscr{L}\left(\mathcal{B}_{\sigma}\right)} \leq \bar{C}_{0} \frac{1}{\sigma^{\alpha+\frac{1}{2}}}\left\|V^{1}-V^{2}\right\|_{\alpha, \sigma}
$$

holds for any $\sigma \geq \sigma_{0}$ with a constant $\bar{C}_{0}$.

Proof. Multiplying $A_{1}[Z]$ in (5.8) by $|p|$ and taking $Z=c / p+V$ we get

$$
\begin{aligned}
|p|\left|\left(A_{1}[Z] B\right)(x, p)\right| & \leq\left(\frac{\left|c_{1}\right|}{|p|^{\frac{3}{2}}}+\frac{|p|^{\alpha}\left|V_{1}(p)\right|}{|p|^{\alpha+\frac{1}{2}}}\right)|p| \int_{0}^{1}|G(x, y, p)| d y \\
& \times\|\nu\|_{C[0,1]}|p| \max _{0 \leq x \leq 1}\left|B_{1}(x, p)\right|+\left(\frac{\left|c_{2}\right|}{|p|^{\frac{3}{2}}}+\frac{|p|^{\alpha}\left|V_{2}(p)\right|}{|p|^{\alpha+\frac{1}{2}}}\right) \\
& \times \sqrt{|p|} \int_{0}^{1}\left|G_{y}(x, y, p)\right| d y\|\mu\|_{C[0,1]}|p| \max _{0 \leq x \leq 1}\left|B_{2}(x, p)\right| .
\end{aligned}
$$

Using Lemma 1 and taking the supremum over $\operatorname{Re} p>\sigma, x \in[0,1]$ we deduce

$$
\begin{aligned}
\left\|A_{1}[Z] B\right\|_{\alpha, \sigma} \leq & \left(\frac{\left|c_{1}\right|}{\sigma^{\frac{3}{2}}}+\frac{\left\|V_{1}\right\|_{\alpha, \sigma}}{\sigma^{\alpha+\frac{1}{2}}}\right) C_{1}\left\|\nu_{k}\right\|_{C[0,1]}\left\|B_{1}\right\|_{1, \sigma} \\
& +\left(\frac{\left|c_{2}\right|}{\sigma^{\frac{3}{2}}}+\frac{\left\|V_{2}\right\|_{\alpha, \sigma}}{\sigma^{\alpha+\frac{1}{2}}}\right) C_{3}\|\mu\|_{C[0,1]}\left\|B_{2}\right\|_{1, \sigma}
\end{aligned}
$$

for $\sigma \geq \sigma_{0}$. This implies

$$
\left\|A_{1}[Z] B\right\|_{\alpha, \sigma} \leq \operatorname{Const}\left(\frac{|c|}{\sigma^{\frac{3}{2}}}+\frac{\|V\|_{\alpha, \sigma}}{\sigma^{\alpha+\frac{1}{2}}}\right)\|B\|_{1, \sigma}, \quad \sigma \geq \sigma_{0} .
$$

For $A_{2}[Z]$ we derive similarly

$$
\begin{aligned}
\sqrt{|p|} \mid & \left(A_{2}[Z] B\right)(x, p)\left|\leq\left(\frac{\left|c_{1}\right|}{|p|^{\frac{3}{2}}}+\frac{|p|^{\alpha}\left|V_{1}(p)\right|}{|p|^{\alpha+\frac{1}{2}}}\right) \sqrt{|p|} \int_{0}^{1}\right| G_{x}(x, y, p) \mid d y \\
& \times\|\nu\|_{C[0,1]}|p| \max _{0 \leq x \leq 1}\left|B_{1}(y, p)\right|+\left(\frac{\left|c_{2}\right|}{|p|^{\frac{3}{2}}}+\frac{|p|^{\alpha}\left|V_{2}(p)\right|}{|p|^{\alpha+\frac{1}{2}}}\right) \\
& \times\left(\frac{1}{\lambda_{0}}+\int_{0}^{1}\left|G_{x y}(x, y, p)\right| d y\right)\|\mu\|_{C[0,1]}|p| \max _{0 \leq x \leq 1}\left|B_{2}(x, p)\right|
\end{aligned}
$$

where $\lambda_{0}=\min _{0 \leq x \leq 1} \lambda(x)>0$. So we get

$$
\left\|A_{2}[Z] B\right\|_{\frac{1}{2}, \sigma} \leq \operatorname{Const}\left(\frac{|c|}{\sigma^{\frac{3}{2}}}+\frac{\|V\|_{\alpha, \sigma}}{\sigma^{\alpha+\frac{1}{2}}}\right)\|B\|_{1, \sigma}, \quad \sigma \geq \sigma_{0}
$$


with some constant. Estimates (6.8) and (6.9) together give us

$$
\|A[Z] B\|_{\sigma} \leq \text { Const }\left(\frac{|c|}{\sigma^{\frac{3}{2}}}+\frac{\|V\|_{\alpha, \sigma}}{\sigma^{\alpha+\frac{1}{2}}}\right)\|B\|_{1, \sigma}, \quad \sigma \geq \sigma_{0} .
$$

This relation shows that $A[Z]$ is well-defined and bounded in $\mathcal{B}_{\sigma}$ and (6.6) holds. Taking $Z=Z_{1}-Z_{2}$ the components of the vector $A[Z]=A\left[Z_{1}\right]-A\left[Z_{2}\right]$ are expressed by the formulas (5.8) where $Z=Z_{1}-Z_{2}=V^{1}-V^{2}$. Using the estimate (6.10) for $A[Z]$ with $c=0$ and $V=V^{1}-V^{2}$ we deduce (5.9). The lemma is proved.

Due to the last two lemmas and the contraction principle, the equation (5.7) has a unique solution $B=B[Z] \in \mathcal{B}_{1, \sigma}$ provided $Z=c / p+V \in \mathcal{M}_{c, \alpha, \sigma}$ and $\sigma \geq \sigma_{0}$ satisfy the relation

$$
\eta(Z, \sigma):=\frac{|c|}{\sigma^{\frac{3}{2}}}+\frac{\|V\|_{\alpha, \sigma}}{\sigma^{\alpha+\frac{1}{2}}} \leq \frac{1}{2 C_{0}} .
$$

From (5.7) we have

$$
\|B[Z]\|_{1, \sigma} \leq\left(1-\|A[Z]\|_{\mathscr{L}\left(\mathcal{B}_{\sigma}\right)}\right)^{-1}\|b[Z]\|_{\sigma} .
$$

This, taking into consideration (6.3), (6.6) and (6.11) gives the estimate for the solution of (5.7)

$$
\|B[Z]\|_{1, \sigma} \leq \operatorname{Const}\left(1+\frac{|c|}{\sqrt{\sigma}}+\frac{\|V\|_{\alpha, \sigma}}{\sigma^{\alpha-\frac{1}{2}}}\right)
$$

with some constant.

Now we will find an estimate for $B\left[Z_{1}\right]-B\left[Z_{2}\right]$. Let $\sigma \geq \sigma_{0}$ and $Z_{1}=$ $c / p+V^{1}, Z_{2}=c / p+V^{2}$ be such that $(6.11)$ is valid, i.e. $\eta\left(Z_{j}, \sigma\right) \leq 1 / 2 C_{0}$, $j=1,2$. Subtracting equation (5.7) for $Z_{2}$ from the corresponding equation for $Z_{1}$ we get

$B\left[Z_{1} 1\right]-B\left[Z_{2}\right]=A\left[Z_{2}\right]\left(B\left[Z_{1}\right]-B\left[Z_{2}\right]\right)+\left(A\left[Z_{1}\right]-A\left[Z_{2}\right]\right) B\left[Z_{1}\right]+b\left[Z_{1}\right]-b\left[Z_{2}\right]$.

This implies

$$
\begin{aligned}
& \mid B\left[Z^{1}\right]-B\left[Z^{2}\right] \|_{1, \sigma} \leq\left(1-\left\|A\left[Z_{2}\right]\right\|_{\mathscr{L}\left(\mathcal{B}_{\sigma}\right)}\right)^{-1} \\
& \quad \times\left[\left\|A\left[Z_{1}\right]-A\left[Z_{2}\right]\right\|_{\mathscr{L}\left(\mathcal{B}_{\sigma}\right)}\left\|B\left[Z_{1}\right]\right\|_{1, \sigma}+\left\|b\left[Z_{1}\right]-b\left[Z_{2}\right]\right\|_{\sigma}\right] .
\end{aligned}
$$

Using in this relation the estimates $(6.2),(6.6),(6.7),(6.11),(6.12)$ we obtain

$$
\left\|B\left[Z_{1}\right]-B\left[Z_{2}\right]\right\|_{1, \sigma} \leq \frac{\text { Const }}{\sigma^{\alpha-\frac{1}{2}}}\left[1+\frac{1}{\sigma}\left(1+\frac{|c|}{\sqrt{\sigma}}+\frac{\left\|V^{1}\right\|_{\alpha, \sigma}}{\sigma^{\alpha-\frac{1}{2}}}\right)\right]\left\|V^{1}-V^{2}\right\|_{\alpha, \sigma} .
$$

Summing up, we have proved the following theorem.

Theorem 1. Let the assumptions (6.1) hold. Then for any $\sigma \geq \sigma_{0}$ and $Z=$ $c / p+V \in \mathcal{M}_{c, \alpha, \sigma}$, satisfying the inequality (6.11), equation (5.7) has a unique solution $B[Z]=\left(B_{1}[Z], B_{2}[Z]\right)$ in $\mathcal{B}_{1, \sigma}$. This solution satisfies estimate $(6.12)$. Moreover, for every $\sigma \geq \sigma_{0}$ and $Z_{1}=c / p+V^{1}, Z_{2}=c / p+V^{2} \in \mathcal{M}_{c, \alpha, \sigma}$ such that $\eta\left(Z^{j}, \sigma\right) \leq 1 / 2 C_{0}, j=1,2$, the difference $B\left[Z_{1}\right]-B\left[Z_{2}\right]$ fulfils estimate (6.13). 


\section{Existence and Uniqueness for Inverse Problem}

Here we study the inverse problem in the fixed-point form (5.4) in the Laplace domain and then formulate the result for the corresponding inverse problem in the time domain.

Theorem 2. Assume that (6.1) holds. Let $\operatorname{det} \Gamma \neq 0$ for $\Gamma$, given by (5.3) and

$$
\Psi_{j}=d_{j} / p+Y_{j} \in \mathcal{M}_{d, \alpha, \sigma_{0}}, \quad j=1,2
$$

with some constant vector $d=\left(d_{1}, d_{2}\right)$. Then there exists $\sigma_{1} \geq \sigma_{0}$ such that equation (5.4) has a unique solution $Z=c / p+V \in \mathcal{M}_{c, \alpha, \sigma_{1}}$. Here $c=\Gamma^{-1} d$. The solution is unique in the union of spaces $\bigcup_{\sigma \geq \sigma_{1}, c \in \mathbb{R}} \mathcal{M}_{c, \alpha, \sigma}$.

Proof. Setting $c=\Gamma^{-1} d$ the problem (5.4) is equivalent to the equation

$$
V=F(V),
$$

where $V \in\left(\mathcal{A}_{\alpha, \sigma}\right)^{2}$ and $F=\Gamma^{-1} \mathcal{F}$. From (5.5), (5.6) we have

$$
\mathcal{F}=L_{0}(c / p+V, B[Z])+L_{1}(c / p+V)+Y,
$$

where $L_{0}$ is a bilinear operator of $Z \in \mathcal{M}_{c, \alpha, \sigma}, B=\left(B_{1}, B_{2}\right) \in \mathcal{B}_{1, \sigma}$ given by

$$
\begin{aligned}
& \left(L_{0}(Z, B)\right)_{k}(p)=\sqrt{p} N(p) \int_{0}^{1} \sqrt{p} G_{x}\left(x_{k}, y, p\right) \nu(y) B_{1}[Z](y, p) d y \\
& -M(p) \int_{0}^{1} p G_{x y}\left(x_{k}, y, p\right) \mu(y) B_{2}[Z](y, p) d y, k=1,2 ; x_{1}=0, x_{2}=1
\end{aligned}
$$

and $L_{1}$ is a linear operator of $Z \in \mathcal{M}_{c, \alpha, \sigma}$ defined by

$$
\begin{aligned}
& \left(L_{1}(Z)\right)_{k}(p)=\sqrt{p} N(p)\left[\int_{0}^{1} \sqrt{p} G_{x}\left(x_{k}, y, p\right) \nu(y) \varphi(y) d y+\frac{(-1)^{k} \nu\left(x_{k}\right) \varphi\left(x_{k}\right)}{\sqrt{\lambda\left(x_{k}\right) \beta\left(x_{k}\right)}}\right] \\
& -M(p)\left[\int_{0}^{1} G_{x y}\left(x_{k}, y, p\right) \mu(y) \varphi^{\prime}(y) d y-\frac{\mu\left(x_{k}\right) \varphi^{\prime}\left(x_{k}\right)}{\lambda\left(x_{k}\right)}\right], k=1,2 ; x_{1}=0, x_{2}=1 .
\end{aligned}
$$

We make use of the fixed-point argument for the equation (7.2) in the balls:

$$
D_{\alpha, \sigma}(\rho)=\left\{V \in\left(\mathcal{A}_{\alpha, \sigma}\right)^{2}:\|V\|_{\alpha, \sigma} \leq \rho\right\} .
$$

First we deduce some estimates for $L_{0}$ and $L_{1}$. Multiplying in (7.4) by $|p|^{\alpha}$ we have

$$
\begin{aligned}
|p|^{\alpha} \mid\left(L _ { 0 } \left(\frac{c}{p}\right.\right. & +V, B))_{k}(p) \mid \leq\left(\frac{\left|c_{1}\right|}{|p|^{2-\alpha}}+\frac{|p|^{\alpha}\left|V_{1}(p)\right|}{|p|}\right) \\
& \times \sqrt{|p|} \int_{0}^{1}\left|G_{x}\left(x_{k}, y, p\right)\right| d y\|\nu\|_{C[0,1]}|p| \max _{0 \leq x \leq 1}\left|B_{1}(x, p)\right| \\
& +\left(\frac{\left|c_{2}\right|}{|p|^{2-\alpha}}+\frac{|p|^{\alpha}\left|V_{2}(p)\right|}{|p|}\right) \int_{0}^{1}\left|G_{x y}\left(x_{k}, y, p\right)\right| d y\|\mu\|_{C[0,1]} \\
& \times|p| \max _{0 \leq x \leq 1}\left|B_{2}(x, p)\right|, \quad k=1,2 ; \quad x_{1}=0, x_{2}=1 .
\end{aligned}
$$


Using the assumptions (6.1) the assertions (4.3), (4.5) of Lemma 1 and the definition of the norms $\|\cdot\|_{\gamma, \sigma}$ we obtain

$$
\begin{gathered}
|p|^{\alpha}\left|\left(L_{0}\left(\frac{c}{p}+V, B\right)\right)_{k}(p)\right| \leq\left(\frac{\left|c_{1}\right|}{|p|^{2-\alpha}}+\frac{\left\|V_{1}\right\|_{\alpha, \sigma}}{|p|}\right) C_{2}\|\nu\|_{C[0,1]}\left\|B_{1}\right\|_{1, \sigma} \\
+\left(\frac{\left|c_{2}\right|}{|p|^{2-\alpha}}+\frac{\left\|V_{2}\right\|_{\alpha, \sigma}}{|p|}\right) C_{4}\|\mu\|_{C[0,1]}\left\|B_{2}\right\|_{1, \sigma}, \quad k=1,2
\end{gathered}
$$

for $\operatorname{Re} p>\sigma, \sigma \geq \sigma_{0}$. Taking the supremum over $\operatorname{Re} p>\sigma$ we get

$$
\left\|L_{0}\left(\frac{c}{p}+V, B\right)\right\|_{\alpha, \sigma} \leq \operatorname{Const}\left(\frac{|c|}{\sigma^{2-\alpha}}+\frac{\|V\|_{\alpha, \sigma}}{\sigma}\right)\|B\|_{1, \sigma}, \quad \sigma \geq \sigma_{0} .
$$

Similarly we deduce for the operator $L_{1}$

$$
\begin{aligned}
& |p|^{\alpha}\left|\left(L_{1}\left(\frac{c}{p}+V, B\right)\right)_{k}(p)\right| \leq\left(\frac{\left|c_{1}\right|}{|p|^{\frac{3}{2}-\alpha}}+\frac{|p|^{\alpha}\left|V_{1}(p)\right|}{\sqrt{|p|}}\right) \sqrt{|p|} \\
& \times\left|\int_{0}^{1} \sqrt{p} G_{x}\left(x_{k}, y, p\right) \nu(y) \varphi(y) d y+\frac{(-1)^{k} \nu\left(x_{k}\right) \varphi\left(x_{k}\right)}{\sqrt{\lambda\left(x_{k}\right) \beta\left(x_{k}\right)}}\right|+\left(\frac{\left|c_{2}\right|}{|p|^{\frac{3}{2}-\alpha}}+\frac{|p|^{\alpha}\left|V_{2}(p)\right|}{\sqrt{|p|}}\right) \\
& \times \sqrt{|p|}\left|\int_{0}^{1} G_{x y}\left(x_{k}, y, p\right) \mu(y) \varphi^{\prime}(y) d y-\frac{\mu\left(x_{k}\right) \varphi^{\prime}\left(x_{k}\right)}{\lambda\left(x_{k}\right)}\right|, \quad k=1,2
\end{aligned}
$$

for $\operatorname{Re} p>\sigma, \sigma \geq \sigma_{0}$. Using Lemma 2 and 3 we get

$$
\begin{gathered}
|p|^{\alpha}\left|\left(L_{1}\left(\frac{c}{p}+V, B\right)\right)_{k}(p)\right| \leq\left(\frac{\left|c_{1}\right|}{|p|^{\frac{3}{2}}-\alpha}+\frac{\left\|V_{1}\right\|_{\alpha, \sigma}}{\sqrt{|p|}}\right) C_{5}\|\nu \cdot \varphi\|_{C[0,1]} \\
+\left(\frac{\left|c_{1}\right|}{|p|^{\frac{3}{2}-\alpha}}+\frac{\left\|V_{2}\right\|_{\alpha, \sigma}}{\sqrt{|p|}}\right) C_{6}\left\|\mu \cdot \varphi^{\prime}\right\|_{C[0,1]} \quad \sigma \geq \bar{\sigma}_{0} .
\end{gathered}
$$

Taking the supremum over $\operatorname{Re} p>\sigma$ we get

$$
\left\|L_{1}\left(\frac{c}{p}+V, B\right)\right\|_{\alpha, \sigma} \leq \operatorname{Const}\left(\frac{|c|}{\sigma^{\frac{3}{2}-\alpha}}+\frac{\|V\|_{\alpha, \sigma}}{\sqrt{\sigma}}\right)\|B\|_{1, \sigma}, \quad \sigma \geq \sigma_{0}
$$

with some constant.

Returning to the equation (7.2) with $F=\Gamma^{-1} \mathcal{F}$, taking into consideration (7.6), (7.7) and the inequality $\|Y\|_{\alpha, \sigma} \leq\|Y\|_{\alpha, \sigma_{0}}$, we obtain

$$
\|\mathcal{F}(V)\|_{\alpha, \sigma} \leq \text { Const }\left(\frac{1}{\sigma^{\frac{3}{2}-\alpha}}+\frac{\|V\|_{\alpha, \sigma}}{\sqrt{\sigma}}\right)\left(\frac{\|B[Z]\|_{1, \sigma}}{\sqrt{\sigma}}+1\right)+\|Y\|_{\alpha, \sigma_{0}}
$$

with $\sigma \geq \bar{\sigma}_{0}$ and some constant depending on $|c|$.

Further, let us suppose that $V \in D_{\alpha, \sigma}(\rho)$, where $\sigma$ and $\rho$ satisfy the relation

$$
\eta_{0}(\rho, \sigma):=\frac{|c|}{\sigma^{\frac{3}{2}}}+\frac{\rho}{\sigma^{\alpha+\frac{1}{2}}} \leq \frac{1}{2 C_{0}}
$$

for $\sigma \geq \sigma_{0}$. Then from $(7.8)$ we see that $\mathcal{F} \in\left(\mathcal{A}_{\alpha, \sigma}\right)^{2}$. Furthermore, due to (7.9) the inequality (6.11) holds, hence we can apply estimate (6.12) of Theorem 1 
for $\|B[Z]\|_{1, \sigma}$. Using this estimate and that $\|V\|_{\alpha, \sigma} \leq \rho$ in the ball $D_{\alpha, \sigma}(\rho)$ we get

$$
\|\mathcal{F}(V)\|_{\alpha, \sigma} \leq \text { Const }\left(\frac{1}{\sigma^{\frac{3}{2}-\alpha}}+\frac{\rho}{\sqrt{\sigma}}\right)\left(1+\frac{1}{\sigma}+\frac{\rho}{\sigma^{\alpha}}\right)+\|Y\|_{\alpha, \sigma_{0}}
$$

with some constant depending on $\rho$ and $\sigma$. From (7.10) due to the equality $F=\Gamma^{-1} \mathcal{F}$ we see that for every $\rho>\rho_{0}:=\left|\Gamma^{-1}\right|\|Y\|_{\alpha, \sigma_{0}}$ there exists $\sigma_{2}=$ $\sigma_{2}(\rho) \geq \bar{\sigma}_{0}$ such that the inequalities $\eta_{0}(\rho, \sigma) \leq \frac{1}{2 C_{0}}$ and $\|F(V)\|_{\alpha, \sigma} \leq \rho$ hold for any $\sigma \geq \sigma_{2}(\rho)$. Consequently,

$$
F: D_{\alpha, \sigma}(\rho) \rightarrow D_{\alpha, \sigma}(\rho) \text { for } \rho>\rho_{0}, \sigma \geq \sigma_{2}(\rho) .
$$

Next, we prove that $F$ is a contraction. From (7.3) using the bilinearity of $L_{0}$ and linearity of $L_{1}$, we have

$$
\begin{aligned}
\mathcal{F}\left(V^{1}\right)-\mathcal{F}\left(V^{2}\right)=L_{0}\left(V^{1}-V^{2}, B\left[Z_{1}\right]\right) & +L_{0}\left(\frac{c}{p}+V^{2}, B\left[Z_{1}\right]-B\left[Z_{2}\right]\right) \\
& +L_{1}\left(V^{1}-V^{2}\right)
\end{aligned}
$$

where $Z_{1}=c / p+V^{1}$ and $Z_{2}=c / p+V^{2}$. Using here (7.6) and (7.7) we get

$$
\begin{aligned}
\left\|\mathcal{F}\left(V^{1}\right)-\mathcal{F}\left(V^{2}\right)\right\|_{\alpha, \sigma} \leq & \text { Const }\left[\frac{\left\|V^{1}-V^{2}\right\|_{\alpha, \sigma}}{\sqrt{\sigma}}\left(\frac{\|B[Z]\|_{1, \sigma}}{\sqrt{\sigma}}+1\right)\right. \\
& \left.+\left(\frac{1}{\sigma^{2-\alpha}}+\frac{\left\|V^{2}\right\|_{\alpha, \sigma}}{\sigma}\right)\left\|B\left[Z_{1}\right]-B\left[Z_{2}\right]\right\|_{1, \sigma}\right], \sigma \geq \sigma_{0}
\end{aligned}
$$

with some constant. Supposing $V^{1}, V^{2} \in D_{\alpha, \sigma}(\rho)$ with $\sigma \geq \sigma_{0}$ and $\rho$ such that (7.9) hold and using the estimates (6.12) and (6.13) of Theorem 1 we obtain

$$
\begin{aligned}
\left\|\mathcal{F}\left(V^{1}\right)-\mathcal{F}\left(V^{2}\right)\right\|_{\alpha, \sigma} \leq & \text { Const }\left[\frac{1}{\sqrt{\sigma}}+\frac{1}{\sigma}+\left(\frac{1}{\sigma^{\frac{3}{2}}}+\frac{\rho}{\sigma^{\alpha+\frac{1}{2}}}\right)\left(2+\frac{1}{\sigma}+\frac{1}{\sigma^{\frac{3}{2}}}+\frac{\rho}{\sigma^{\alpha+\frac{1}{2}}}\right)\right] \\
& \times\left\|V^{1}-V^{2}\right\|_{\alpha, \sigma} .
\end{aligned}
$$

The coefficient of $\left\|V^{1}-V^{2}\right\|_{\alpha, \sigma}$ on the right-hand side of this estimate approaches zero as $\sigma \rightarrow \infty$ for a fixed $\rho>0$. Hence, for every $\rho>0$ there exists $\sigma_{3}=\sigma_{3}(\rho) \geq \bar{\sigma}_{0}$, such that the inequality $\eta_{0}(\rho, \sigma) \leq \frac{1}{2 C_{0}}$ holds and $F=\Gamma^{-1} \mathcal{F}$ is a contraction in the ball $D_{\alpha, \sigma}(\rho)$ for $\rho>0$ and $\sigma \geq \sigma_{3}(\rho)$. This together with (7.11) shows that equation (7.2) has a unique solution $V$ in every ball $D_{\alpha, \sigma}(\rho)$, where $\rho>\rho_{0}$ and $\sigma \geq \sigma_{4}(\rho)=\max \left\{\sigma_{2}(\rho) ; \sigma_{3}(\rho)\right\}$. This proves the existence assertion with $\sigma_{1}=\sigma_{4}\left(2 \rho_{0}\right)$.

To prove uniqueness we show that the equation (5.4) has no solution in any space in the whole space $\mathcal{M}_{\bar{c}, \alpha, \sigma}$ where $\bar{c} \neq c=\Gamma^{-1} d$ and $\sigma \geq \sigma_{1}$. Suppose contrary that (5.4) has a solution $Z=\bar{c} / p+V$ in some of such spaces.Then observing (5.5), (7.1) we see that component $V \in\left(\mathcal{A}_{\alpha, \sigma}\right)^{2}$ of this solution satisfies the equation

$$
\Gamma V=L_{0}\left(\frac{c}{p}+V, B[Z]\right)+L_{1}\left(\frac{c}{p}+V\right)+Y+\frac{\bar{c}-c}{p},
$$


where $L_{0}$ and $L_{1}$ are given by (7.4), (7.5). Observing the assumption (7.1) and the estimates $(7.6),(7.7)$ we see that all terms in (7.12) except the last one $(\bar{c}-c) / p$ are estimated by quantities of the form Const $/|p|^{\alpha}$ in the half-plane $\operatorname{Re} p>\sigma_{0}$, where the constant is independent of $p$ and $\alpha>1$. This is not possible in the case $\bar{c}-c \neq 0$. We reached the contradiction. The uniqueness is proved.

Finally, we deduce from Theorem 2 the existence and uniqueness for the initial inverse problem in the time domain. From

$$
Z_{1}(p)=\sqrt{p} N(p)=\frac{c_{1}}{p}+V_{1}(p)
$$

we get image of the kernel of the internal energy $N(p)=c_{1} / p^{\frac{3}{2}}+\widetilde{V}_{1}(p)$. Using the inverse Laplace transform we obtain the following result.

Theorem 3. Let the assumptions (6.1) hold and $\operatorname{det} \Gamma \neq 0$ for $\Gamma$, given by (5.3). Then the inverse problem (2.1)-(2.3), (2.5), (2.6) with flux observations in the time domain has the unique solution

$$
n(t)=\frac{2 c_{1}}{\sqrt{\pi}} \sqrt{t}+\frac{1}{2 \pi i} \int_{\xi-i \infty}^{\xi+i \infty} e^{t p} \widetilde{V}_{1}(p) d p, \quad m(t)=c_{2}+\frac{1}{2 \pi i} \int_{\xi-i \infty}^{\xi+i \infty} e^{t p} V_{2}(p) d p
$$

where $c_{k} \in \mathbb{R}, V_{k} \in \mathcal{A}_{\alpha, \sigma}, k=1,2$. The functions $n(t)$ and $m(t)$ are continuous for $t \geq 0$. Moreover, the vector $c=\left(c_{1}, c_{2}\right)$ in the formulas (7.13) is expressed by $c=\Gamma^{-1} d$, where $d$ is component of $\Psi$ in the assumption (7.1) and $c_{2}=m(0)$.

\section{References}

[1] B.D. Coleman and M.E. Gurtin. Equipresence and constitutive equation for rigid heat conduction. Z. Angew. Math. Phys., 18:199-208, 1967. Doi:10.1007/BF01596912.

[2] M. Grasselli. An identification problem for a linear integrodifferential equation occurring in heat flow. Math. Meth. Appl. Sci., 15:167-186, 1992. Doi:10.1002/mma.1670150304.

[3] J. Janno. Determination of a time- and space-dependent heat flux relaxation function by means of a restricted Dirichlet-to-Neumann operator. Math. Meth. Appl. Sci., 27:1241-1260, 2004. Doi:10.1002/mma.490.

[4] J. Janno and L. v. Wolfersdorf. An inverse problem for identification of a timeand space-dependent memory kernel of a special kind in heat conduction. Inverse Problems, 15:1455-1467, 1999. Doi:10.1088/0266-5611/15/6/305.

[5] J. Janno and L. v. Wolfersdorf. Identification of a special class of memory kernels in one-dimensional heat flow. J. Inv. Ill-Posed Problems, 9:389-411, 2001.

[6] J. Janno and L. v. Wolfersdorf. Identification of memory kernels in onedimensional heat flow with boundary conditions of the third kind. Inverse Problems in Engineering, 9:179-198, 2001. Doi:10.1080/174159701088027760.

[7] A. Lunardi. On the linear equation with fading memory. SIAM J. Math. Anal., 21:1213-1224, 1970. Doi:10.1137/0521066. 
[8] J. W. Nunziato. On heat conduction in materials with memory. Quart. Appl. Math., 29:187-204, 1971.

[9] E. Pais. Degenerate memory kernels identification problem with fluxtype additional conditions. J. Inv. Ill-Posed Problems, 14:397-418, 2006. Doi:10.1515/156939406777571003.

[10] E. Pais and J. Janno. Identification of two degenerate time- and space-dependent kernels in a parabolic equation. Electron. J. Differ. Eqns., 2005(108):1-20, 2005.

[11] E. Pais and J. Janno. Inverse problem to determine degenerate memory kernels in heat flux with third kind boundary conditions. Math. Model. Anal., 11:427-450, 2006.

[12] E. Pais and J. Janno. Recovering Memory Kernels in Heat Flow. Inverse Problems for Non-Homogeneous Degenerate Memory Kernels. Lambert Academic Publishing, Köln, 2009.

[13] I.G. Petrowski. Vorlesungen über partielle Differentialgleichungen. Teubner, Leipzig, 1955. (in German) 